\title{
The reddest ISO-2MASS quasar ${ }^{\star}$
}

\author{
C. Leipski ${ }^{1, \star \star}$, M. Haas ${ }^{1}$, R. Siebenmorgen ${ }^{2}$, H. Meusinger ${ }^{3}$, M. Albrecht ${ }^{4}$, C. Cesarsky ${ }^{2}$, \\ R. Chini ${ }^{1}$, R. Cutri ${ }^{5}$, H. Drass ${ }^{1}$, J. P. Huchra ${ }^{6}$, S. Ott ${ }^{7}$, and B. J. Wilkes ${ }^{6}$
}

\author{
1 Astronomisches Institut Ruhr-Universität Bochum (AIRUB), Universitätsstraße 150, 44780 Bochum, Germany \\ e-mail: leipski@physics.ucsb.edu \\ 2 European Southern Observatory (ESO), Karl-Schwarzschild-Str. 2, 85748 Garching, Germany \\ Thüringer Landessternwarte Tautenburg (TLS), Sternwarte 5, 07778 Tautenburg, Germany \\ ${ }^{4}$ Instituto de Astronomía, Universidad Católica del Norte (UCN), Avenida Angamos 0610, Antofagasta, Chile \\ 5 IPAC, California Institute of Technology (Caltech), 770 South Wilson Avenue, Pasadena, CA 91125, USA \\ ${ }^{6}$ Harvard-Smithsonian Center for Astrophysics (CfA), 60 Garden Street, Cambridge, MA 02138, USA \\ 7 HERSCHEL Science Centre, ESA, Noordwijk, PO Box 299, 2200 AG Noordwijk, The Netherlands
}

Received 30 August 2006 / Accepted 11 July 2007

\section{ABSTRACT}

\begin{abstract}
Context. While there is growing consensus on the existence of numerous dust-enshrouded red quasars, their discovery and detailed exploration is still an observational challenge. In the course of the near-mid-infrared AGN search combining the 6.7 $\mu \mathrm{m}$ ISOCAM Parallel Survey and 2MASS we have discovered 24 type-1 quasars about a third of which are too red to be discriminated by optical/UV search techniques.

Aims. Here we report on a detailed case study of the reddest type-1 quasar of our sample, 2MASS J23410667-0914327 (for short J2341) at redshift $z=0.236$ with $M_{K}=-25.8$ and $J-K_{\mathrm{s}}=1.95$. This source has a very red optical appearance but lacks the far-infrared emission typically seen in known dust enshrouded AGN. Therefore we here explore its enigmatic nature.

Methods. We performed spectroscopy in the optical with VLT/FORS1 and in the mid-infrared (5-38 $\mu \mathrm{m})$ with the Spitzer Space Telescope as well as near-infrared (NIR) imaging with ISPI at the CTIO $4 \mathrm{~m}$-telescope. To explain the red optical continuum of the quasar we examine nuclear dust reddening of an intrinsically blue quasar continuum in combination with dilution by stellar light of the host galaxy.

Results. The optical and NIR observations reveal a star forming emission-line galaxy at the same redshift as the quasar with a projected linear separation of 1".8 $(6.7 \mathrm{kpc})$. The quasar and its companion are embedded in diffuse extended continuum emission. Compared with its companion the quasar exhibits redder optical-NIR colours, which we attribute to hot nuclear dust. The mid-infrared spectrum shows only few emission lines superimposed on a power-law spectral energy distribution typically seen in type-1 AGN. However, the lack of strong far-infrared emission suggests that our potentially interacting object contains much less gas and dust and is in a stage different from dust reddened ULIRG-AGN like Mrk 231. The optical spectrum shows signatures for reddening in the emission-lines and no post-starburst stellar population is detected in the host galaxy of the quasar. The optical continuum emission of the active nucleus appears absorbed and diluted.

Conclusions. Even the combination of absorption and host dilution is not able to match J2341 with standard quasar templates. While the BLR shows only a rather moderate absorption of $E_{B-V}=0.3$, the continuum shorter than $4500 \AA$ A requires strong obscuration with $E_{B-V}=0.7$, clearly exceeding the constraints from the low upper limit on the silicate $9.7 \mu \mathrm{m}$ absorption. This leads us to conclude that the continuum of the quasar $\mathbf{J} 2341$ is intrinsically redder than that of typical quasars.
\end{abstract}

Key words. galaxies: active - quasars: general - infrared: galaxies

\section{Introduction}

Pure optical quasar surveys find QSOs with essentially blue continua (e.g. Schmidt \& Green 1983; Wolf 2005). However, investigations including the radio, $\mathrm{X}$-ray and infrared have revealed a substantial fraction of dust-reddened AGN missed by optical surveys (Low et al. 1988; Gregg et al. 2002; Maiolino et al. 2003; Glikman et al. 2004; Lacy et al. 2004). We have combined the

* Based on observations made with ESO Telescopes at La Silla and Paranal under programme IDs 275.A-5064 and 075.A-0374, with the Spitzer Space Telescope, which is operated by the JPL, CALTECH under a contract with NASA, and obtained at CTIO a division of NOAO, which is operated by AURA under cooperative agreement with the National Science Foundation.

$\star \star$ Present address: University of California, Santa Barbara, CA-93106, USA.
ISOCAM Parallel Survey at $6.7 \mu \mathrm{m}$ (Siebenmorgen et al. 1996) with the 2MASS survey (Skrutskie et al. 2006) in order to obtain a powerful tool to search for AGN by means of their infrared colours $\left(H-K_{\mathrm{s}}>0.5\right.$ and $K_{\mathrm{s}}-L W 2(6.7 \mu \mathrm{m})>2.7$; Haas et al. 2004). In fact, in a high galactic latitude area of $\sim 10 \mathrm{deg}^{2}$ we found $30 \%$ more type-1 quasars per square degree down to $R=18$ mag than e.g. the SDSS DR3 quasar survey (Leipski et al. 2005). The quasars found by our NIR/MIR method show a variety of spectral shapes, in particular in the optical. Some of the ISO-2MASS objects are too red to be recognised as quasars by current optical strategies, probably because of dust extinction. Since the NIR and MIR colours of quasars are different from those of stars, infrared selection enables the detection of optically red quasars (Cutri et al. 2002; Smith et al. 2003; Leipski et al. 2005; Lacy et al. 2007). 
From our sample of 77 AGN candidates 24 turned out to be type-1 AGN. They span a range of optical colours from blue quasar-typical ones to very red ones similar to those found in dust-enshrouded ULIRG-AGN. The reddest quasar of our sample is 2MASS J23410667-0914327 (hereafter called J2341) at a moderate redshift $z=0.236$. Thus, its red photometric appearance is not due to high redshift.

Alternatively to the classical $M_{B}<-23$ mag quasar definition, its absolute $K_{\mathrm{s}}$-band brightness of $M_{K_{\mathrm{s}}}=-25.8 \mathrm{mag}$ qualifies $\mathbf{J} 2341$ as a QSO. The absolute SDSS $i$ magnitude of $M_{i}=$ $-23.0 \mathrm{mag}$ and an [O III] $\lambda 5007$ luminosity of $L_{[\mathrm{O} \mathrm{III}]} \sim 2.7 \times$ $10^{41} \mathrm{erg} \mathrm{s}^{-1}$ places $\mathrm{J} 2341$ at the low luminosity end of the total luminosity distribution of quasars found in the SDSS, but at intermediate values for objects in the $z=0.2-0.3$ regime (Schneider et al. 2007). While no radio counterpart is listed by NVSS (translating to $F_{1.4 \mathrm{GHz}}<2.5 \mathrm{mJy}$; Condon et al. 1998), ROSAT HRI observations give a soft X-ray luminosity of $L_{0.5-2.4 \mathrm{keV}} \sim 3 \times 10^{43} \mathrm{erg} \mathrm{s}^{-1}$, similar to that of low redshift PG-quasars. The galactic foreground extinction is $E_{B-V}=0.03$ (Schlegel et al. 1998).

The low IRAS $60 \mu \mathrm{m}$ upper limit $F_{60 \mu \mathrm{m}}<129 \mathrm{mJy}$ suggests that $\mathbf{J} 2341$ is different from known ULIRG-AGN. If this is true, then its red optical colours are puzzling. Therefore we performed a detailed case study of $\mathbf{J} 2341$, using optical VLT spectra, nearinfrared imaging from the CTIO $4 \mathrm{~m}$ telescope and mid-infrared spectra from the Spitzer Space Telescope.

\section{Observations}

The quasar nature of J2341 was discovered on spectra taken with EMMI at the ESO/NTT telescope during the optical follow-up spectroscopy of our MIR selected AGN candidates. We used a 1 '. 5 wide long slit oriented along the parallactic angle (slit-loss effects due to atmospheric dispersion are minimised by such a slit orientation) and centred on the peak of the apparent brightness distribution (Fig. 1). These observations were obtained before the object was recognised as a double source. The slit width in combination with Grism\#2 results in a spectral resolution of $\sim 600 \mathrm{~km} \mathrm{~s}^{-1}$. The integration time was $3 \times 20 \mathrm{~min}$.

In order to check morphological details and the NIR photometry, we have obtained $J H K_{\mathrm{s}}$ images with ISPI at the CTIO $4 \mathrm{~m}$-Blanco telescope. These observations were performed under stable seeing conditions $\left(F W H M \sim 1^{\prime \prime}\right)$ with a total on-source integration time of $15 \mathrm{~min}$ in each filter. The images were reduced using standard IRAF procedures.

After detecting that J2341 may be a double source, we have further obtained optical spectra with VLT/FORS1 in order to explore the nature of $\mathrm{J} 2341$ and its putative companion. The 1 .' 3 wide slit was oriented to cover both objects (Fig. 1) and an atmospheric dispersion corrector was used for the $1 \times 15$ min observation. In combination with grism GRIS_300V the observations yield a spectral resolution of $\sim 650 \mathrm{~km} \mathrm{~s}^{-1}$.

The optical spectra were reduced using standard procedures within the ESO/MIDAS package. Since no spatial structure could be identified on the 2D spectroscopic frames from the NTT run we combined all the flux into a single spectrum. This procedure may include contributions from the underlying host galaxy and from the companion, diluting the QSO signatures in the final NTT spectrum. The spectroscopic observations with the VLT were able to spatially resolve both objects since the slit was optimally oriented. We searched for the brightest spectral column in broad line flux and in continuum for the QSO and the companion, respectively. We extracted 3-pixel wide columns $\left(0.2^{\prime \prime} / \mathrm{px}\right)$ into single spectra, each corresponding to only 0.6 spatially.

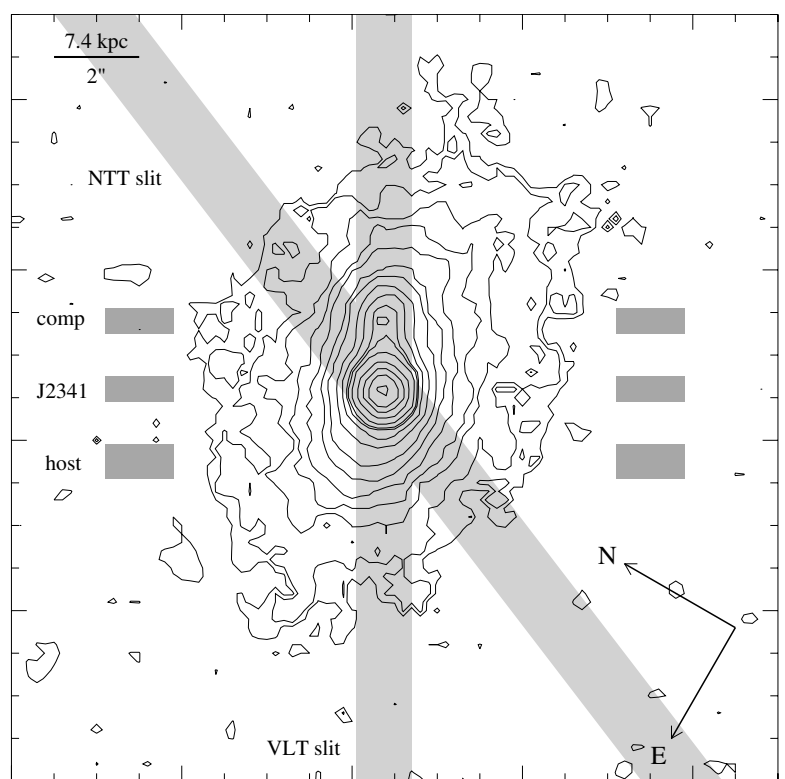

Fig. 1. Contour plot of the J2341 "system" from the $30 \mathrm{~s}$ VLT $R$-band acquisition image. The contour levels have been chosen to highlight the extended emission. The image shows $18^{\prime \prime} \times 18^{\prime \prime}(90 \mathrm{px} \times 90 \mathrm{px})$. The positions and widths of the VLT slit (p.a. $120^{\circ} ; 1^{\prime \prime} .3$ width) and the NTT slit (p.a. $155^{\circ} ; 1^{\prime \prime} .5$ width) are indicated by the lightly shaded rectangles. The darker shaded areas indicate the positions and widths of the extraction regions for the spectra used in this paper.

Since the seeing was $\sim 11^{\prime \prime} 0$ during the observations this choice reduces the flux in the extracted spectra but minimises the contributions from the adjacent source while providing adequate $\mathrm{S} / \mathrm{N}$ ratios.

We also obtained a low-resolution 5-38 $\mu \mathrm{m}$ MIR spectrum of J2341 using IRS (Houck et al. 2004) aboard Spitzer Space Telescope (Werner et al. 2004) with total integration times of $240 \mathrm{~s}$ and $480 \mathrm{~s}$ for SL and LL, respectively. We used data processed by version S15.3.0 of the IRS pipeline and performed calibration and extraction of the spectra within SPICE.

\section{Comparison of the NTT and VLT spectra}

Here we briefly consider whether observational effects or host galaxy contamination is important for the red appearance of the quasar spectrum and how far the quasar and companion spectra may be contaminated by each other.

In Fig. 2 we plot the NTT spectrum (extracted through a large aperture) over the VLT spectrum of the QSO (extracted through a narrow aperture). The shape of the continuum agrees very well for both observations. We conclude that the red continuum is intrinsic to the source and not an artifact from the data reduction or due to slit-loss effects. In addition, the red optical continuum is also evident from USNO and SDSS photometry (e.g. Fig. 3 in Leipski et al. 2005).

Compared with the VLT spectrum, the NTT spectrum includes more flux from the underlying host galaxy and potentially some flux from the companion as well. Therefore it shows more pronounced and even additional stellar absorption lines. The increased continuum flux also results in smaller equivalent widths (EWs) of the emission lines (Fig. 2).

Despite the stronger stellar contribution in the NTT spectrum, the shape of the continuum is the same for both the VLT and NTT spectra. This suggests that the influence of stellar light contributions on the shape of the observed continuum can be 


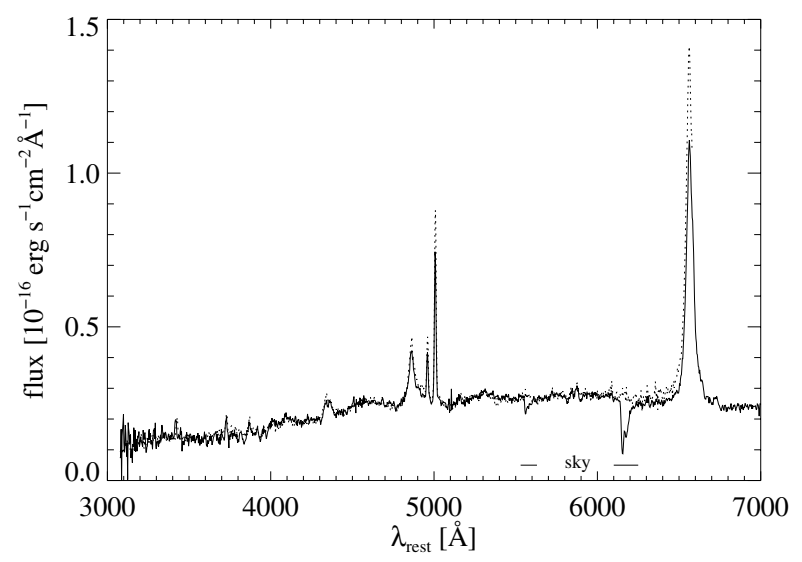

Fig. 2. Comparison of the VLT spectrum (dotted line) and the NTT spectrum (solid line; scaled to match the continuum between 4000 and $6000 \AA)$.

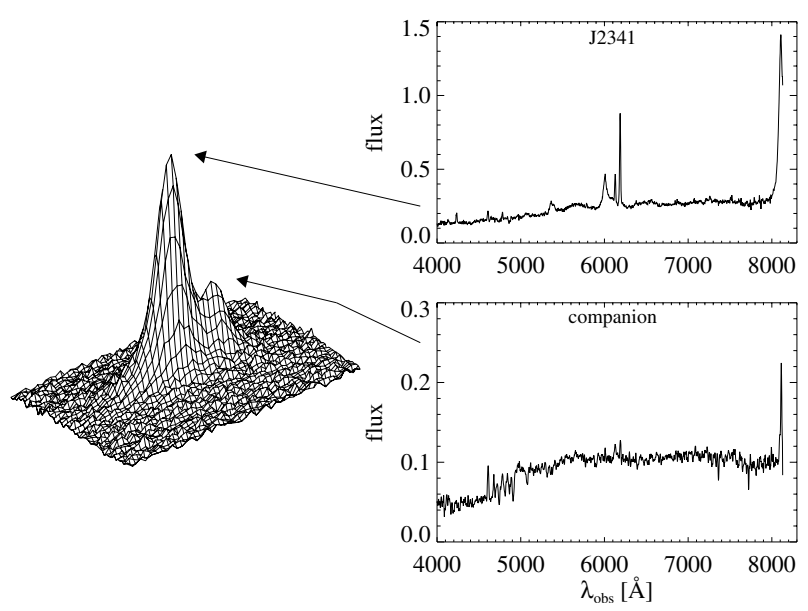

Fig. 3. $R$-band surface plot and spectra of the two objects obtained with FORS1/VLT. The flux is given in $0^{-16} \mathrm{erg} \mathrm{s}^{-1} \mathrm{~cm}^{-2} \AA^{-1}$.

significant even for the nuclear VLT spectrum. We will address the underlying host galaxy further in Sect. 4.4.2.

In Figs. 3 and 4 we show the VLT spectra of the QSO and its companion. These spectra were extracted through narrow apertures to minimise contaminations from each other. The absence of broad emission-line components and emission from the highly ionised inner parts of the narrow-line region (e.g. [Ne V]) in the spectrum of the companion strongly argues against significant contributions from emission of the QSO. The QSO spectrum does not show signs for higher order Balmer absorption lines which are on the other hand prominent in the companion. This supports our conclusion that the spectra of the QSO and the companion show the intrinsic features of the respective source and that they are not significantly contaminated by each other.

\section{Results and discussion}

The inspection of the $J H K_{\mathrm{s}}$ frames and the $R$-band acquisition image (30 s integration time) reveals a nearby faint object approximately 1'.8 north-west of J2341 (Figs. 1 and 3; position angle $294 \mathrm{deg}$ ), which could be a physically interacting companion galaxy. The VLT spectra reveal that both objects lie at the same redshift $(z=0.236)$. This and the small projected separation of 1 .' $8(\sim 6.7 \mathrm{kpc})$ suggests that they are in fact interacting. This is further supported by recent and ongoing star

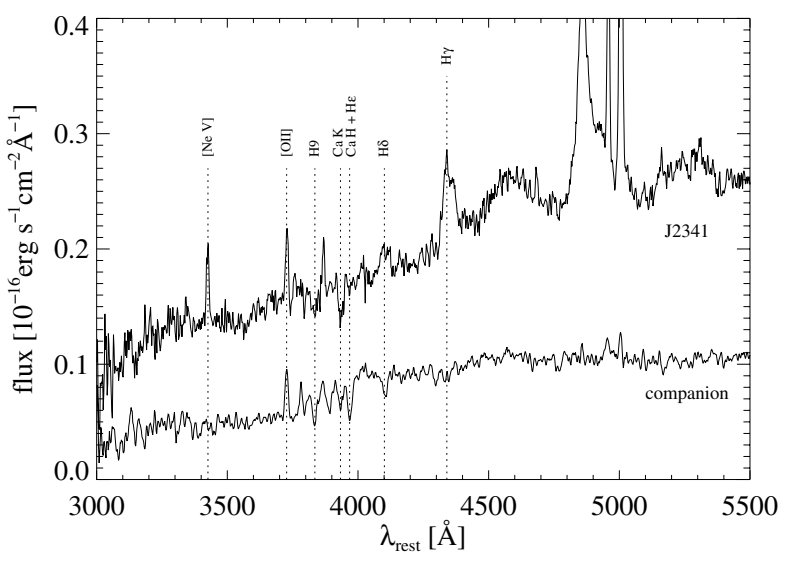

Fig. 4. Blue portion of the spectra of the quasar and its companion.

formation in the companion (Sect. 4.2). The images do not show clear morphological peculiarities or tidal tails but both galaxies are embedded in weak extended emission which is best seen in the $R$-band image (Fig. 1). In order to assess the amount of extended emission we subtracted two Gaussian point sources, one for the QSO and one for the companion. The Gaussian width was determined from nearby stars and fits their profiles as well as that of the QSO flux peak. Table 1 lists the resulting photometry for the entire system as well as the flux ratios QSO/companion and nuclear/total. Our analysis shows that the contribution of the extended emission is largest in $R$ and $J$.

In the following discussion, the name "J2341" will refer to the quasar and the secondary object is called "companion".

\subsection{The quasar}

The spectrum of $\mathrm{J} 2341$ shows broad Balmer emission lines $\left(F W H M \sim 2800 \mathrm{~km} \mathrm{~s}^{-1}\right.$, rest frame) and a red optical continuum (Fig. 2). This is unusual for a type-1 AGN. We just note that some unusual broad absorption line QSOs (BALQSOs) show very red SEDs due to wide, overlapping BAL troughs in the ultraviolet (e.g. Hall et al. 2002; Meusinger et al. 2005). While the continuum of J2341 is smooth and does not exhibit any indications for BAL structures over the wavelength range covered by our spectra, the presence of BALs at $\lambda<3000 \AA$ cannot be excluded. It is well known that BALQSOs tend to be more dust reddened than non-BALQSOs (Reichard et al. 2003).

Figure 5 displays the mean SDSS quasar template (Vanden Berk et al. 2001) over the spectrum of J2341. While J2341 agrees with the optical quasar template at $\lambda>5500 \AA$, it is redder shortwards of that wavelength where the continuum of classical quasars is dominated by blue, power-law like emission. J2341 is also redder than reddened quasars usually found using optical multi-colour selection techniques (e.g. Richards et al. 2003). The flux ratio of the broad Balmer emission lines is larger for J2341 than for the template, indicating that the BLR of J2341 is also reddened. We will address the reddening further in Sect. 4.4.1.

Figure 4 shows an enlarged view of the blue portion of the quasar spectrum. Broad Balmer emission lines and Fe II emission are detected, as well as low and high ionisation forbidden lines from the narrow-line region (e.g. [Ne V], [O II], [Ne III], [O III]). We also detect $\mathrm{Ca}$ II $\mathrm{K}$ as an absorption feature from the host galaxy. The presence of Ca II K and the absence of higher order Balmer absorption suggests that J2341 does not 


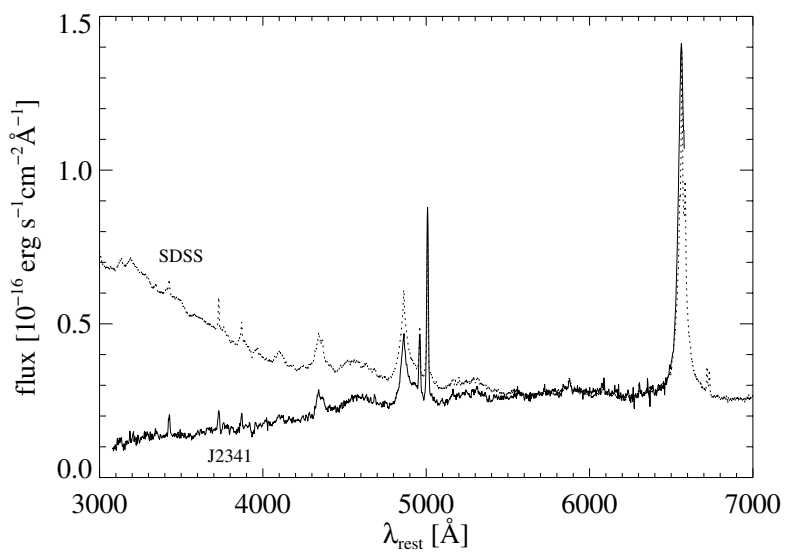

Fig. 5. Spectra of J2341 and the mean SDSS quasar template of Vanden Berk et al. (2001). The SDSS spectrum was scaled to match $\mathrm{J} 2341$ in $\mathrm{H} \alpha$.

have a strong contribution from a young-to-intermediate age stellar population $(<1 \mathrm{Gyr})$.

In the mid infrared (Fig. 6) J2341 shows a power-law continuum typical for classical AGN (e.g. Weedman et al. 2005; Ogle et al. 2006; Buchanan et al. 2006). We see the [O IV] emission line at the 5- $\sigma$ level. The high ionisation $[\mathrm{Ne} \mathrm{V}]$ lines are not detected in the MIR, although prominent in the optical spectrum (Fig. 4). We also do not detect the low ionisation emission lines [Ne II] and [Ne III]. The detection of [O IV] and the upper limits on the $[\mathrm{Ne} \mathrm{V}]$ lines are consistent with the fact that in AGN [O IV] is usually the brightest MIR line, on average about three times brighter than the [Ne V] lines (e.g. Haas et al. 2005). On the other hand, weak PAH emission features at $7.7 \mu \mathrm{m}$ and $11.3 \mu \mathrm{m}$ are seen. Albeit of marginal significance, they are present at both nod positions of the spectral frames.

The slits of IRS $\left(\sim 3\right.$ 3. $^{\prime} 6$ and $\sim 10$.' 6 for SL and LL, respectively) include the quasar as well as its companion, but the MIR continuum is likely to be dominated by dust emission from the quasar itself, which is already 5.5 times brighter at $K_{\mathrm{s}}$ than the companion (Table 1).

With $v L_{\gamma}(15 \mu \mathrm{m})=2.1 \times 10^{44} \mathrm{erg} / \mathrm{s}$ the MIR luminosity of $\mathbf{J} 2341$ is relatively low compared to powerful AGN but the source can still be considered MIR strong (Ogle et al. 2006). J2341 turns out to be almost a factor of 10 less luminous in the MIR than type-2 AGN at similar redshift which were found in the ISO-2MASS survey (Leipski et al. 2007).

It is expected that the interaction of both objects triggers enhanced nuclear star formation, when gas and dust are concentrated in the central regions. To study the rate of star formation in quasars, Ho (2005) examined the [O II $] /[\mathrm{O}$ III] flux ratio of PG quasars. Low ratios between $10 \%$ and $30 \%$ are consistent with AGN origin. Using this method for $\mathbf{J} 2341$ the low [O II $] /[\mathrm{O}$ III] ratio of $\approx 10 \%$ does not indicate strong ongoing star formation.

In Fig. 6 we overplot the IRS spectrum of the broad-line AGN/ULIRG Mrk 231 (Weedman et al. 2005). In contrast to J2341, Mrk 231 shows silicate absorption, although broad permitted lines are present in its optical spectrum. In J2341 on the other hand it seems that there is not enough dust between the putative torus (as the source of the MIR continuum emission) and the observer to produce significant silicate absorption. Mrk 231 also exhibits a significant FIR excess that indicates powerful hidden starbursts. J2341 has relatively low FIR emission as derived from IRAS-ADDSCANs $\left(F_{60 \mu \mathrm{m}}<129 \mathrm{mJy}\right)$ and from the flat 15-30 $\mu \mathrm{m}$ slope of the MIR spectrum (Fig. 6). Therefore, dusty

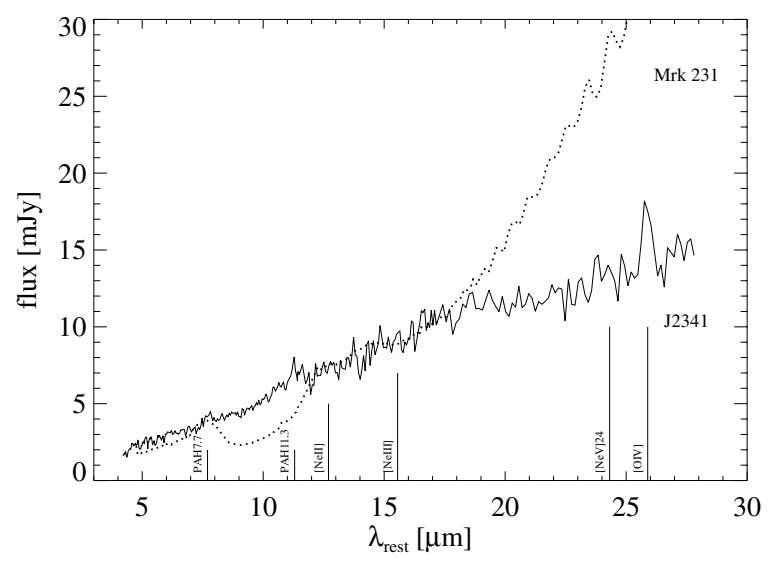

Fig. 6. IRS/Spitzer spectra of J2341 and Mrk 231 scaled to match J2341 at $15 \mu \mathrm{m}$. The positions of important emission lines are marked.

starbursts as found in Mrk 231 are not present, as already inferred from the optical spectrum. We suggest that in J2341 the total amount of dust and gas for fuelling starbursts, which can be triggered by the interaction, is low.

\subsection{The companion}

The spectrum of the companion is reminiscent of an e(c) galaxy with rather strong $\mathrm{H} \delta$ absorption (Dressler et al. 1999). As a result of a starburst in the past a significant population of A type stars exist in the companion as traced by prominent Balmer absorption lines (Fig. 4). We can speculate that the first encounter of this galaxy with the quasar has initiated a burst of star formation in the companion resulting in the strong population of A stars observed today.

Ongoing star formation is traced by emission lines like [O II] and $\mathrm{H} \alpha$. This star formation is likely to be triggered by the interaction. The observed $\mathrm{H} \alpha$ emission could in principle be a contamination from an extended narrow-line region of the quasar. However, the low observed [O III] flux and the very different $[\mathrm{O}$ II $] /[\mathrm{O}$ III $]$ flux ratio suggests that most of the line emission is intrinsic to the companion. While the $\mathrm{H} \alpha / \mathrm{H} \beta$ ratio appears to be large suggesting significant reddening, the $\mathrm{H} \beta$ line in this object is affected by strong stellar Balmer absorption masking the intrinsic reddening.

The absence of strong FIR emission as evident from the Spitzer spectrum at $20-30 \mu \mathrm{m}$ (Fig. 6) suggests that there is no strong, dust-enshrouded starburst in the companion, which could contribute to the FIR flux. Since ongoing star formation is weak in the host galaxy of the quasar but clearly observed in the companion, most of the PAH features detected in the MIR spectrum could actually arise in the companion.

\subsection{Comparison with other red quasars}

Our ISPI NIR photometry reveals that for our slightly extended system some flux is missed when measured via the (default) point-source photometry of 2MASS (Table 1). More appropriate is the 2MASS aperture photometry (4"aperture), which includes both objects. It agrees well with our new photometry which in turn reduces the $J-K_{\mathrm{s}}$ colour of the quasar alone from 1.95 to $\sim 1.5$ (Table 1). The flux ratio of $\mathbf{J} 2341$ to its companion is similar in $R, J$, and $H$, while it significantly different in $K_{\mathrm{s}}$. This indicates that both objects are dominated by a comparable old stellar population at red optical to NIR wavelengths. In this case their 
Table 1. Photometry of the J2341 system, given in Vega magnitudes. The errors of the NIR photometry are $\leq 0.1 \mathrm{mag}$. (2) Default 2 MASS PSF photometry includes both objects. The $R$-mag is taken from USNO-B. (3) 2MASS 4"aperture photometry. (4) ISPI aperture photometry, $R$-mag taken from the VLT acquisition frame. (5) Peak flux ratio of quasar-to-companion from nuclear (1") Gaussian fitting. (6) Fraction of total flux contained in nuclear Gaussian fits (quasar + companion); the complementary flux is due to extended emission.

\begin{tabular}{cccccc}
\hline \hline $\begin{array}{c}\text { Filter } \\
(1)\end{array}$ & $\begin{array}{c}\text { 2MASS } \\
(2)\end{array}$ & $\begin{array}{c}\text { 2MASS } \\
(3)\end{array}$ & $\begin{array}{c}\text { ISPI } \\
(4)\end{array}$ & $\begin{array}{c}\text { QSO/comp } \\
(5)\end{array}$ & $\begin{array}{c}\text { nuclear/total } \\
(6)\end{array}$ \\
\hline$R$ & 17.52 & - & 18.02 & 3.0 & 0.34 \\
$J$ & 16.505 & 16.065 & 16.01 & 3.1 & 0.35 \\
$H$ & 15.502 & 15.234 & 15.21 & 3.3 & 0.42 \\
$K_{\mathrm{S}}$ & 14.555 & 14.577 & 14.49 & 5.5 & 0.44 \\
\hline
\end{tabular}

$R-K_{\mathrm{S}}$ colour should also be the same. We attribute the $0.6 \mathrm{mag}$ increase in $R-K_{\mathrm{s}}$ in the quasar to additional hot dust emission. This also indicates that the central regions of the quasar are not severely obscured which otherwise would affect the NIR emission.

The $J-K_{\mathrm{S}}$ colour of $\mathrm{J} 2341$ is bluer than the threshold used to select the 2MASS red AGN $\left(J-K_{\mathrm{s}}>2\right.$; Cutri et al. 2002) which show various optical continuum slopes (e.g. Smith et al. 2003) and X-ray properties (e.g. Wilkes et al. 2002). For the 2MASS red AGN we most likely observe a range from essentially unobscured to absorbed sources. The red $J-K_{\mathrm{s}}$ colour can then be attributed to either clearly visible emission from hot dust in the centre or to absorption of the $J$ continuum.

In the picture where nuclear activity is triggered by interaction red quasars are supposed to represent young members of the QSO population. While so far only a moderate fraction of 2MASS red AGN and classical blue PG quasars show signs for interaction on HST images ( 30\%; Marble et al. 2003), J2341 clearly has a spectroscopically confirmed companion (Fig. 3).

\subsection{On the origin of the red quasar continuum}

We now discuss the faint optical continuum emission of J2341, which is unusual for optically selected type-1 QSOs. The aim is to see how far common mechanisms are able to explain the nature of $\mathrm{J} 2341$.

First, we assume that J2341 has an intrinsically blue continuum similar to the SDSS quasar template from Vanden Berk et al. (2001), but that $\mathbf{J} 2341$ is reddened by a dust screen (Sect. 4.4.1) and in addition diluted by stellar light of the host galaxy (Sect. 4.4.2).

It should be noted that the SDSS template is quite similar to other QSO composites like those from the Large Bright Quasar Survey or the First Bright Quasar Survey (Fig. 11 in Vanden Berk et al. 2001). The relative spectrum-to-spectrum variation of the quasars forming the SDSS template is about $15-20 \%$ in the wavelength range 3000-5000 $\AA$ (Fig. 10 in Vanden Berk et al. 2001), hence relatively small. The power-law slope between Ly $\alpha$ and $\mathrm{H} \beta$ is somewhat redder for a composite formed from $z<0.84$ quasars $\left(\alpha_{v}=-0.65 ; S \sim v^{\alpha}\right)$ than the slope for the entire quasar sample entering the SDSS template $\left(\alpha_{v}=-0.44\right)$. But this effect is small for the wavelength range considered here, i.e. compared with template from the entire sample, the redder slope of the $z<0.84$ template reduces the $3000 \AA$ continuum by only $\sim 10 \%$, when keeping the $4863 \AA$ flux fixed. Therefore, we here use the SDSS template from the entire sample, assuming that it represents an essentially unreddened quasar spectrum. Furthermore, the SDSS template still contains contributions from the host galaxy (especially at red optical wavelengths), hence for a reasonable comparison any host contributions in J2341 will rather tend to make the differences to the SDSS template smaller than larger.

Since these two mechanisms - dust screen reddening and dilution by the host galaxy - do not fully explain the red nature of $\mathrm{J} 2341$, we discuss further scenarios in Sect. 4.4.3.

\subsubsection{Dust screen reddening}

In numerous cases it has been shown that the spectra of luminous red quasars can be matched with template spectra quite well if simple dust screen reddening is applied (e.g. quasars from the FIRST-2MASS survey, Gregg et al. 2002). This technique works also well for low- $z$ AGN where host galaxy contributions become important. For example, one nearby red type-1 QSO from the Spitzer First Look Survey (SSTXFLS J171335.1+584756 at $z=0.133$; Lacy et al. 2007) shares some basic properties with J2341: FLS J1713 has a red optical continuum, broad Balmer emission lines (although narrower than in J2341), and Ca II absorption lines indicating contributions from the host galaxy. However, between $24 \mu \mathrm{m}$ and $70 \mu \mathrm{m}$ the SED of FLS J1713 looks more like that of the ULIRG-AGN Mrk231, with a $70 \mu \mathrm{m} / 24 \mu \mathrm{m}$ flux ratio of about 4 . As a test we reddened the SDSS template using SMC dust extinction curves (Gordon et al. 2003) to match the spectrum of FLS J1713. The rest-frame continuum is decently represented between $\sim 4000 \AA$ and $8000 \AA$ by the SDSS template reddened with $E_{B-V}=0.5$ (shortwards of $\lambda_{\text {rest }}=4000 \AA$ no spectral data are available). This test demonstrates that even some nearby red quasars (which include host galaxy emission) can be explained by simple screen reddening of optical QSO templates, at least longward of $\lambda_{\text {rest }}=4000 \AA$.

Figure 5 shows the spectrum of $\mathrm{J} 2341$ and the unreddened SDSS QSO template. The spectra are scaled to match in the $\mathrm{H} \alpha$ line. This highlights the difference in the optical continua of J2341 and the template. It suggests reddening in J2341 bluewards of $5500 \AA$ which becomes particularly strong for $\lambda_{\text {rest }}<$ $4000 \AA$.

After applying a dust screen reddening of $E_{B-V}=0.3$ to the template both spectra match well down to $\sim 4500 \AA$ (Fig. 7, top). Also, the fluxes of the broad $\mathrm{H} \alpha$ and $\mathrm{H} \beta$ lines match for both spectra. This shows that a dust screen of $E_{B-V}=0.3$ accounts pretty well for reddening on sightlines towards the broad-line region of $\mathbf{J} 2341$ (assuming the same intrinsic Balmer ratio). We determined the uncertainty of the spectral matching to $\Delta E_{B-V} \sim$ 0.02 .

However, shortwards of $4500 \AA \mathrm{J} 2341$ still lacks significant continuum flux compared to the reddened template (Fig. 7, top). At $3000 \AA$ the discrepancy is about a factor 3 . An $E_{B-V}=0.7$ would be necessary to match the blue part of the template with the observed $\mathbf{J} 2341$ spectrum (Fig. 7, bottom). But with $E_{B-V}=$ 0.3 we already have accounted for the amount of dust towards the centre as traced by the broad emission lines. If a reddening of $E_{B-V}=0.7$ was indeed present towards the central regions of J2341 the intrinsic $\mathrm{H} \alpha / \mathrm{H} \beta$ would be $\sim 2$ which is not supported by either theory (e.g. Kwan 1984) or observations (e.g. Shang et al. 2007). Therefore, other explanations than extinction by a dust screen have to be considered to account for the remaining difference at blue optical wavelengths. 

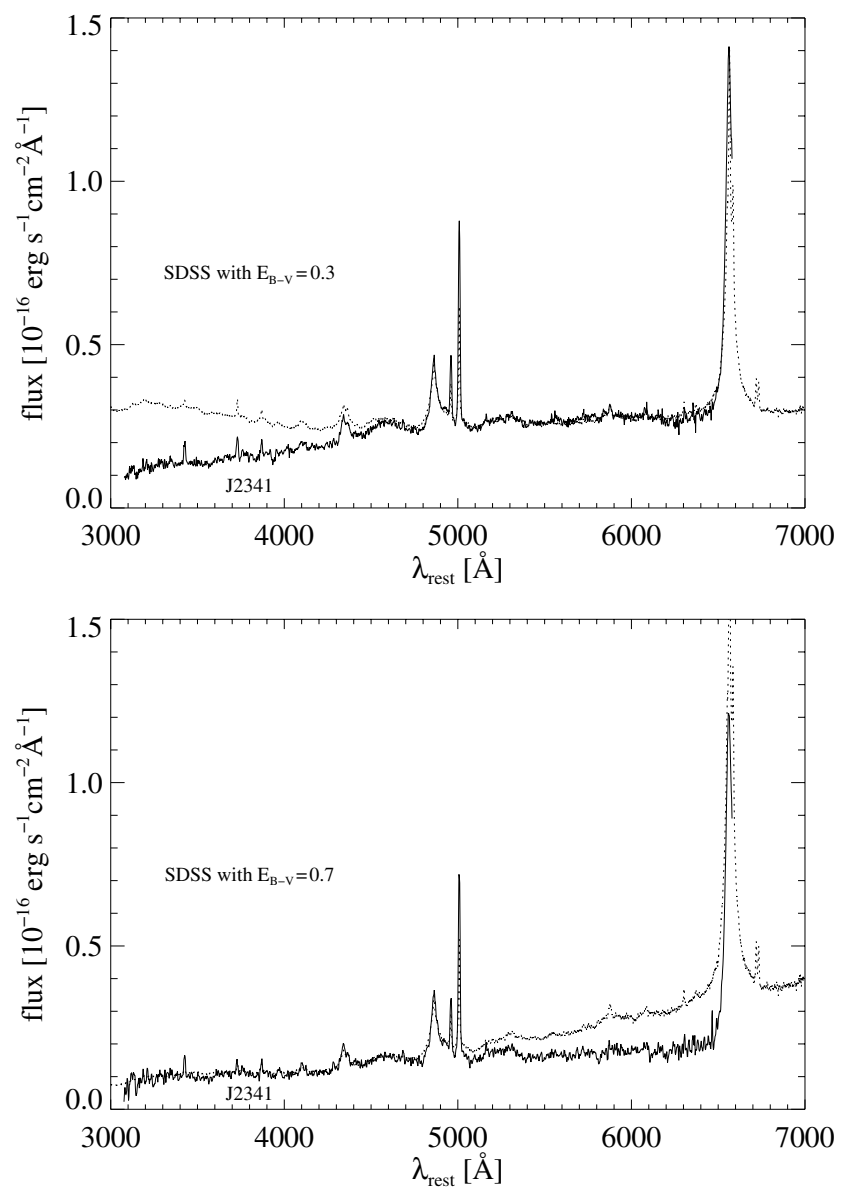

Fig. 7. Comparison of the spectrum of J2341 with the SDSS quasar template moderately reddened $E_{B-V}=0.3$ (top), and strongly reddened $E_{B-V}=0.7$ (bottom).

\subsubsection{Dilution by host galaxy light}

An underlying host galaxy with a pronounced $4000 \AA$ A break can in principle dilute the signature of the blue bump emission, i.e. the rising blue continuum.

We note that QSO templates - especially at red optical wavelengths - are dominated by emission from local, lower luminosity AGN and, thus, a significant host galaxy component may already be included (e.g. Vanden Berk et al. 2001; Gaskell et al. 2004). On the other hand, for blue optical wavelengths higher redshift (and higher luminosity) sources dominate the template and the influence of the host galaxy on the overall spectral shape of the template is reduced.

Since the subtraction of several template galaxy spectra taken from the literature (e.g Calzetti et al. 1994; Kinney et al. 1996) give poor results we therefore extracted the host galaxy spectrum directly from our two dimensional spectral frames (see also Bennert et al. 2006). This results in a much better fit to the absorption features in the QSO, even considering the moderate $\mathrm{S} / \mathrm{N}$ of the host spectrum.

The host spectrum was extracted from an area of 0.8 with a separation of $1^{\prime \prime}$ south-east $(3.7 \mathrm{kpc})$ to the region of the QSO spectrum (Fig. 1). The placement and width of the region used for the host spectrum extraction was chosen to provide maximal $\mathrm{S} / \mathrm{N}$ without contamination from nuclear QSO light. The host spectrum shows clear $\mathrm{Ca}$ II $\mathrm{H}+\mathrm{K}$ absorption as well as [O II] and narrow $\mathrm{H} \alpha /[\mathrm{N}$ II $]$ emission (Fig. 8, top). For the same reasons as for the companion spectrum we expect the stellar template to be
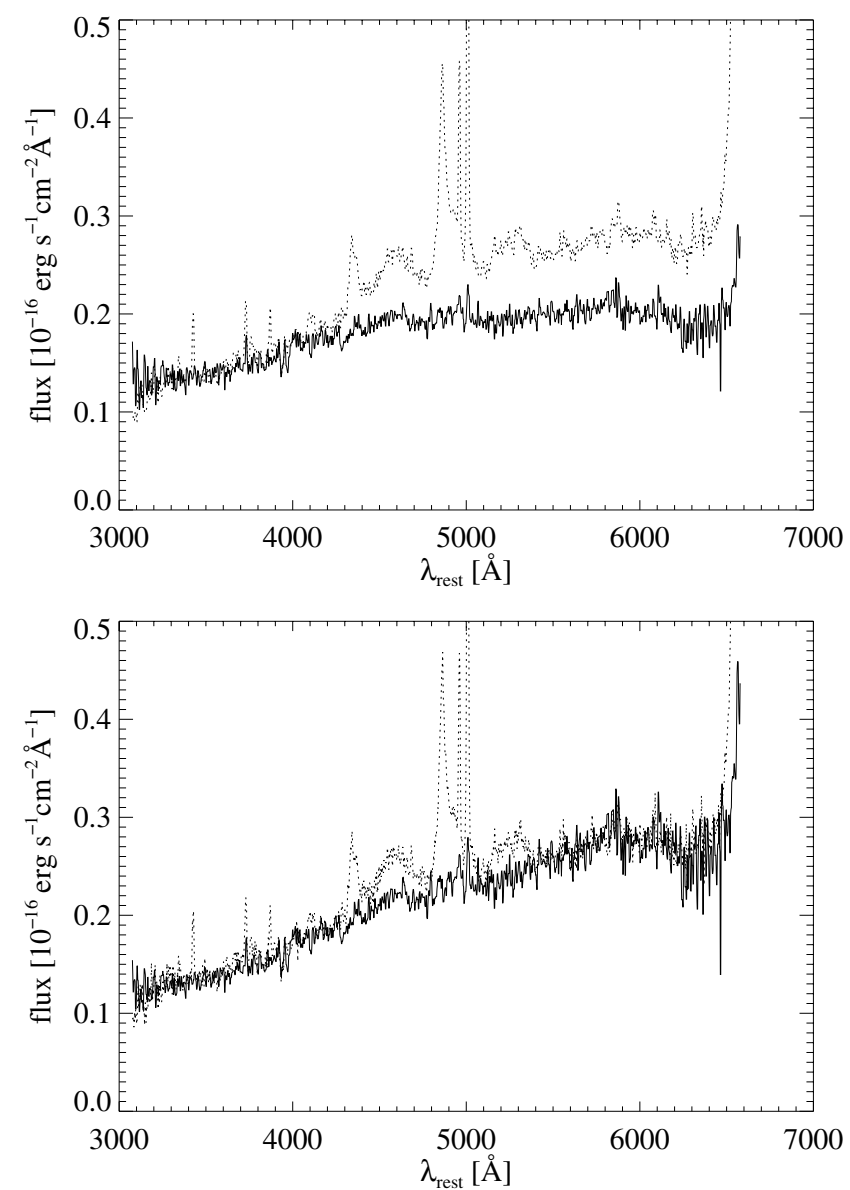

Fig. 8. The extracted host spectrum without (top) and with additional reddening of $E_{B-V}=0.3$ ontop of the QSO spectrum (dashed line). The host spectra are scaled to remove the Ca II K feature from the QSO after subtraction. Just for these graphs additional offsets were applied to the host spectra to facilitate an easy comparison with the QSO.

largely free of AGN contamination (no broad-line components, no high-ionisation lines; see Sect. 3). While the [O II], $\mathrm{H} \alpha$, and the weak [O III] emission could result from an extended narrowline region, it can also indicate ongoing star formation in the host galaxy. The flux ratio of $F_{\text {[O II] }} \geq F_{\text {[O III] }}$ suggests star formation as cause for the line emission in the host galaxy.

Following Boroson et al. (1982) we also obtained a host galaxy spectrum by subtracting the nuclear 0 .' $^{\prime} 6$ spectrum from a spectrum of 3". 0 width which covered the quasar plus host emission (on the opposite side from the companion). The nuclear spectrum was scaled in such a way that the broad lines were removed and only the underlying host galaxy spectrum remained. This spectrum agrees very well with the spectrum from the off-nuclear extraction supporting our approach. In the further analysis only the off-nuclear spectrum was used.

We scaled the host spectrum in such a way that, after subtraction from the QSO spectrum, the Ca II K line is removed. We also tried other scalings but even the most extreme values explored were not able to recover the blue bump emission while on the other hand highly overestimating the Ca II K absorption. Thus, we used the Ca II K line as a measure for the host galaxy contribution.

This subtraction also recovers the $\mathrm{H} \epsilon$ line in a reasonable strength (Fig. 9, top). This line was largely absent in the original spectrum due to the underlying $\mathrm{Ca}$ II $\mathrm{H}$ absorption. Down to 

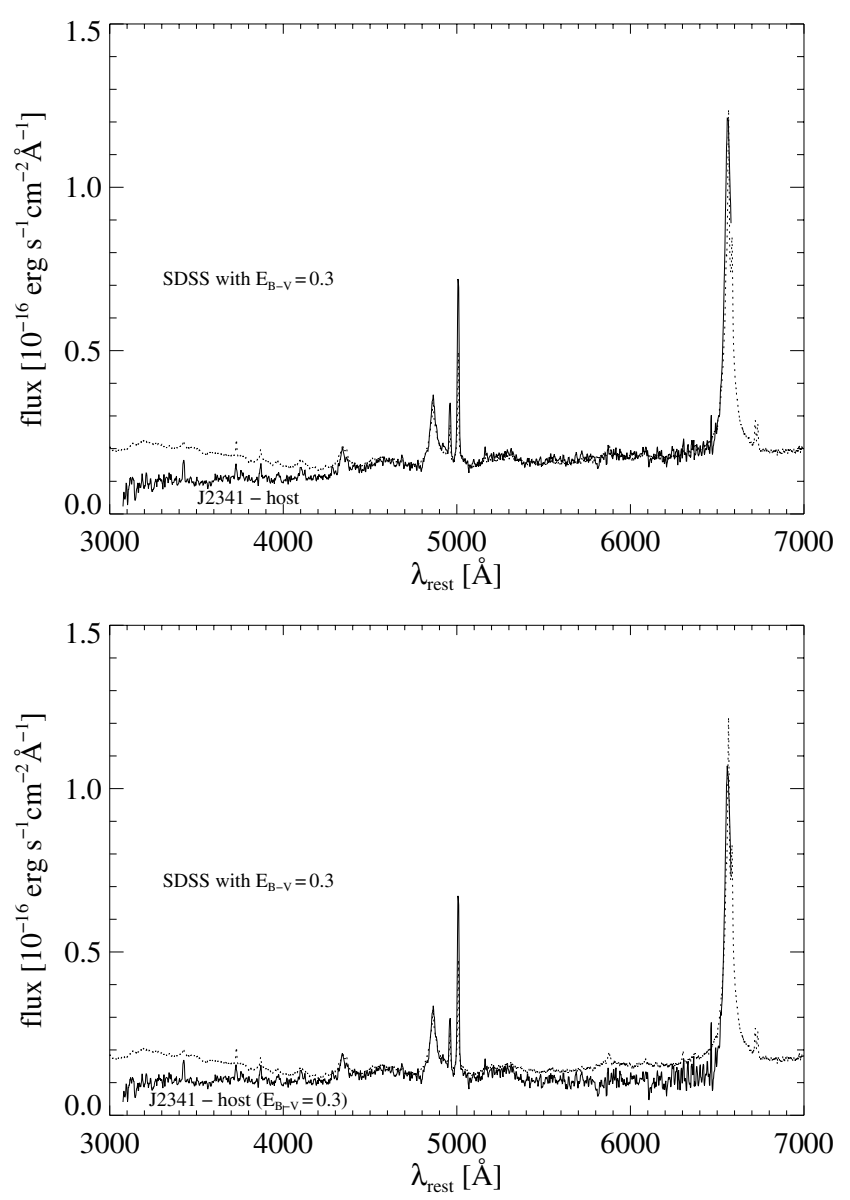

Fig. 9. Comparison of the moderately reddened SDSS quasar template with the spectrum of J2341 after subtraction of the host without reddening (top), and with additional reddening (bottom).

about $4500 \AA$ the resulting "host-free" QSO spectrum of J2341 fits the dust reddened SDSS QSO template better than the original J2341 spectrum does. But shortwards of $4500 \AA$ the "hostfree" J2341 system still lacks continuum, and at $3000 \AA$ the discrepancy is about a factor of 2 . This is too large to be explained by the 10-20\% spectrum-to-spectrum variation of the SDSS template or the $10 \%$ redder slope of the $z<0.84$ template. Even if a redder template made of extraordinarily UVweak quasars would fit $\mathbf{J} 2341$ better, it is of particular interest to explore the nature of such potentially UV-weak quasars.

A refined way to account for the remaining discrepancies between the QSO template and the J2341 spectrum would be to consider increased reddening towards the centre of the system, as found by e.g. Bennert et al. (2006) for their Seyfert galaxies. Then the nuclear host galaxy component, as seen in the J2341 QSO spectrum, would be more reddened than the extracted host galaxy template $3.7 \mathrm{kpc}$ further out. Reddening of the host galaxy spectrum before subtraction from the QSO gives an interesting results (Fig. 8, bottom): After applying a redding equivalent to $E_{B-V}=0.3$ the reddened host galaxy spectrum follows the spectral shape of the QSO remarkably closely. (We chose $E_{B-V}=0.3$ since that was the value inferred as reddening towards the broad-line region.) Due to the similarity in spectral shape the subtraction of the reddened host galaxy spectrum from the QSO results in the QSO spectrum being almost flat in flux over the observed wavelengths (Fig. 9, bottom). This makes the fit of the J2341 spectrum with the SDSS QSO template worse over almost the whole wavelength range compared to the case with an unreddened host galaxy.

It is also possible that the stellar population in the centre differs from that at a few kiloparsec further out. But Bennert et al. (2006) found this issue to be only of minor importance for their spatially well resolved object (NGC 1386).

To summarise, so far the comparison with the SDSS quasar template strongly suggest that dust screen reddening alone and the combination of screen reddening with host galaxy contributions are not able to fully explain the red optical/UV continuum of $\mathrm{J} 2341$ shortwards of $\mathrm{H} \beta$. Therefore, we consider alternative scenarios.

\subsubsection{Refined scenarios and alternatives}

In this section we discuss special dust distributions and other effects. So far we have applied a dust screen of $E_{B-V}=0.3$ to the SDSS template in order to match its spectrum with J2341 after subtraction of the host. The dust cannot be distributed evenly throughout the whole galaxy, because we clearly detect features of the host galaxy in the optical spectrum of the quasar, while the AGN continuum appears heavily suppressed (Fig. 4). Moreover, the emission lines from the NLR are still clearly visible in J2341 suggesting that the major part of the obscuring material is located in the centre of the galaxy, somewhere between the NLR and the accretion disc (e.g. Fig. 4).

Small, sharply confined dust clouds with a low volume filling factor may lie by chance in our line of sight towards the accretion disk, which produces the continuum emission. Then the clouds may redden the AGN continuum emission, while the bulk of the slightly more extended BLR may be less affected. This scenario appears in accordance with results from X-ray spectroscopy of red 2MASS type-1 AGN, where the presence of a compact absorber very near to the active nucleus has been proposed. In these AGN the BLR is clearly visible, but the X-ray source is significantly obscured (e.g. Wilkes et al. 2005). We should note, however, that the X-ray absorption traces the gas while the optical obscuration is due to dust. In addition, if a low volume filling factor is assumed, the swirling dust clouds are expected to shield the centre not permanently (transient shielding). Hence, we expect significant variability in both the total intensity and the spectral shape. But we detect neither a significant difference in the spectral slope of our spectra (that span two years) nor are significant photometric differences observed by comparison of SDSS and USNO-B data (that span 40 years).

Another explanation considers that light from the nucleus grazes the edge of a moderately inclined torus so that the BLR is largely seen, but the accretion disk is essentially hidden and strongly reddened (see also Smith et al. (2004) who proposed a similar scenario to explain the polarisation properties of a group of Seyfert 1 galaxies). Then the torus must have a rather sharp edge, a requirement which looks unlikely because the ongoing interaction favours a more disturbed dust distribution. In addition, the MIR spectra do not show recognisable silicate absorption placing an upper limit on the MIR absorption and, thus, on the optical absorption of $A_{\mathrm{V}}<1$ (following Krügel et al. 2003). This upper limit is consistent with our finding of $E_{B-V}=0.3$. Therefore even this special geometry does not provide a satisfying explanation for substantially higher extinction towards the accretion disk.

Next we discuss screen versus mixed case reddening. Simple screen reddening by dust is not suitable to explain simultaneously the reddening of the BLR and the continuum. But dust mixed with the BLR may be in accordance with the observations. 
Then the BLR suffers only moderate mixed case reddening, while the continuum is more strongly reddened by the entire absorbing screen. However, this requires dust inside the dust sublimation radius mixed with the BLR, where it should ultimately be destroyed by the strong radiation field of the nucleus.

Since common mechanisms fail to provide a satisfying explanation for the red continuum of $\mathrm{J} 2341$, it seems that the strong Blue Bump emission of average quasars is largely missing in J2341. For low-luminosity AGN (LLAGN) radiatively inefficient accretion flows (RIAFs) were proposed to explain the lack of prominent Big Blue Bump emission in the UV (e.g. Ho 1999; Narayan 2005). Although J2341 qualifies as a bona fide quasar from its luminosities at different wavelengths, the spectral shape towards the UV is reminiscent of RIAFs. Since the dust models have difficulties to consistently explain the spectrum of J2341, the RIAF scenario is an attractive possibility to interpret the observations. However, the detection of prominent high excitation lines like $[\mathrm{Ne} \mathrm{V}]$ strongly questions an intrinsically low UV flux.

Even the refined scenarios have difficulties matching J2341 with standard blue quasar templates. This leads us to suggest that the continuum of the quasar J2341 is intrinsically redder than that of typical quasars, although not being in a RIAF phase.

Since the enigmatic UV-weak nature of J2341 may also be found in other objects further studies of this prototypical object are desired.

Higher $\mathrm{S} / \mathrm{N}$ data in which several stellar absorption features are securely identified could allow for a more detailed way to account for the host galaxy contribution. On the other hand, observations in the rest-frame UV range would be especially interesting for the apparent lack of a prominent Small Blue Bump while strong Balmer and Fe II emission is observed in the optical. Studies sampling this wavelength range are needed to further address this phenomenon.

Acknowledgements. Part of this work was supported by Sonderforschungsbereich SFB 591 "Universelles Verhalten gleich-gewichtsferner Plasmen" der Deutschen Forschungsgemeinschaft, and by Nordrhein-Westfälische Akademie der Wissenschaften. We thank Dan Weedman for kindly providing the Spitzer spectrum of Mrk 231. We also thank Andy Robinson for his helpful comments on the manuscript. Based on observations with the Infrared Space Observatory ISO, an ESA project with instruments funded by ESA Member States (especially the PI countries: France, Germany, the Netherlands and UK) and with the participation of ISAS and NASA. The Two Micron All Sky Survey is a joint project of the University of Massachusetts and IPAC/Caltech, funded by the National Aeronautics and Space Administration and the National Science Foundation.

\section{References}

Antonucci, R. R. J. 1993, ARA\&A, 31, 473

Bennert, N., Jungwiert, B., Komossa, S., et al. 2006, A\&A, 446, 919

Boroson, T. A., Oke, J. B., \& Green, R. F. 1982, ApJ, 263, 32

Buchanan, C. L., Gallimore, J. F., O’Dea, C. P., et al. 2006, AJ, 132, 401

Calzetti, D., Kinney, A. L., \& Storchi-Bergmann, T. 1994, ApJ, 429, 582

Condon, J. J., Cotton, W. D., Greisen, E. W., et al. 1998, AJ, 115, 1693

Cutri, R., Nelson, B., Francis, P., \& Smith, P. 2002, ASP 284, 127

Dressler, A., Smail, I., Poggianti, B. M., et al. 1999, ApJS, 122, 51

Gaskell, C. M., Goosmann, R. W., Antonucci, R. R. J., \& Whysong, D. H. 2004, ApJ, 616, 147

Glikman, E., Gregg, M. D., Lacy, M., et al. 2004, ApJ, 607, 60

Gregg, M. D., Lacy, M., White, R. L., et al. 2002, ApJ, 564, 133

Gordon, K. D., Clayton, G. C., Misselt, K. A., et al. 2003, ApJ, 594, 279

Haas, M., Siebenmorgen, R., Leipski, C., et al. 2004, A\&A, 419, L49

Haas M., Siebenmorgen R., Schulz B., et al. 2005, A\&A, 442, L39

Hall, P. B., Anderson, S. F., Strauss, M. A, et al. 2002, ApJS, 141, 267

Ho, L. C. 1999, ApJ, 516, 672

Ho, L. C. 2005, ApJ, 629, 680

Houck, J. R., Roellig, T. L., van Cleve, J., et al. 2004, ApJS, 154, 18

Kinney, A. L., Calzetti, D., Bohlin, R. C., et al. 1996, ApJ, 467, 38

Krügel, E. 2003. The Physics of Interstellar Dust (Bristol: Institute of Physics Publishing)

Kwan, J. 1984, ApJ, 283, 70

Lacy, M., Storrie-Lombardi, L., Sajina, A., et al. 2004, ApJS, 154, 166

Lacy, M., Petric, A. O., Sajina, A., et al. 2007, AJ, 133, 186

Leipski, C., Haas, M., Meusinger, H., et al. 2005, A\&A, 440, L8

Leipski, C., Haas, M., Meusinger, H., et al. 2007, A\&A, 464, 895

Low, F., Cutri, R., Huchra, J., \& Kleinmann, S. 1988, ApJ, 327, L41

Maiolino, R., Comastri, A., Gilli, R., et al. 2003, MNRAS, 344, L59

Marble, A. R., Hines, D. C., Schmidt, G. D., et al. 2003, ApJ, 590, 707

Meusinger, H., Froebrich, D., Haas, M., et al. 2005, A\&A, 433, L25

Narayan, R. 2005, Ap\&SS, 300, 177

Ogle, P., Whysong, D., \& Antonucci, R. 2006, ApJ, 647, 161

Papovich, C., Cool, R., Eisenstein, D., et al. 2006, AJ, 132, 231

Reichard, T. A., Richards, G. T., Schneider, D. P., et al. 2003, AJ, 125, 1711

Richards, G. T., Hall, P. B., Vanden Berk, D. E., et al. 2003, 126, 1131

Schlegel, D. J., Finkbeiner, D. P., \& Davis, M. 1998, ApJ, 500, 525

Schmidt, M., \& Green, R. F. 1983, ApJ, 269, 352

Schneider, D. P., Hall, P. B., Richards, G. T., et al. 2007, [arXiv: 0704 .0806]

Shang, Z., Wills, B. J., Wills, D., \& Brotherton, M. S. 2007, [arXiv:astro-ph/0703690]

Siebenmorgen, R., Abergel, A., et al. 1996, A\&A, 315, L169

Skrutskie, M. F., Cutri, R. M., Stiening, R., et al. 2006, AJ, 131, 1163

Smith, P. S., Schmidt, G. D., Hines, D. C., \& Foltz, C. B. 2003, ApJ, 593, 676

Smith, J. E., Robinosn, A., Alexander, D. M., et al. 2004, MNRAS, 350, 140

Vanden Berk, D. E., Richards, G. T., Bauer, A., et al. 2001, AJ, 122, 549

Weedman, D. W., Hao, L., Higdon, S. J. U., et al. 2005, ApJ, 633, 706

Werner, M. W., Roellig, T. L., Low, F. J., et al. 2004, ApJS, 154, 1

Wilkes, B. J., Schmidt, G. D., Cutri, R. M., et al. 2002, ApJ, 564, L65

Wilkes, B. J., Pounds, K. A., Schmidt, G. D., et al. 2005, ApJ, 634, 183

Wolf, C., 2005, MmSAI, 76, 21 